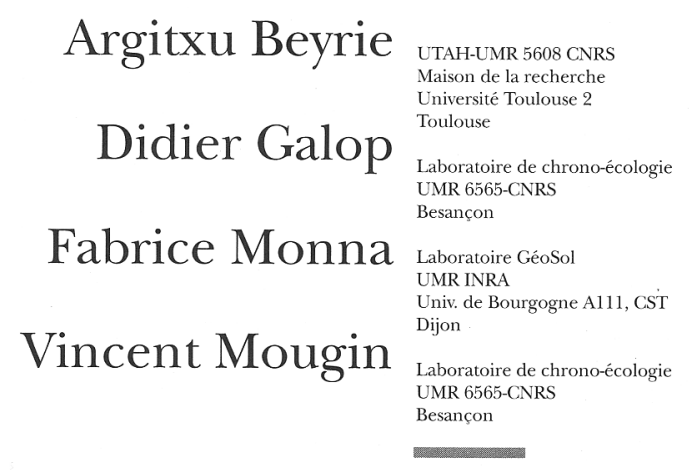

\title{
La métallurgie du fer au Pays Basque durant l'Antiquité. État des connaissances dans la vallée de Baigor ri (Pyrénées-Atlantiques)
}

\section{RÉSUMÉ}

Une étude de la métallurgie antique du fer a été engagée au Pays Basque, dans la vallée de Baigorri, un secteur dont les gisements métallifères ont été exploités dès avant l'Antiquité et parfois jusqu'au Xxe siècle. Les prospections et les investigations entreprises ont révélé l'existence de deux districts miniers et métallurgiques dont la chronologie est comprise entre la fin de l'âge du Fer (II siècle a.C.) et le IV siècle p.C. Les mines, les charbonnières et les sites métallurgiques des deux centres de production ont été identifiés, tandis que quatre ateliers de réduction ont fait l'objet d'une étude archéologique particulière. Ces résultats ont été confrontés aux données paléoenvironnementales issues de l'analyse palynologique et géochimique d'une tourbière de la haute vallée de Baigorri.

\begin{abstract}
A study of the ancient metallurgy of iron was committed in the Basque Country, in the Baigorri valley, a rich sector whose resources were exploited before Antiquity and sometimes until the century $\mathrm{xx}^{\text {th }}$. The prospecting and the investigations undertaken revealed the existence of two mining and metallurgical districts. Their chronology is attested between the end of the Iron age and the century IVth $p . C$. The mines, the coal-scuttles and the metallurgic sites of the two centres of production were identified, while four workshops of reduction were the subject of a particular archaeological study. These results were confronted with the environmental data resulting from the palynological and geochemical analysis of a peat bog from the high valley of Baigorri.
\end{abstract}

\section{MOTS-CLÉS}

Mine, métallurgie, histoire de l'environnement, Pyrénées, fer, Antiquité 
L'extrémité occidentale de la chaîne pyrénéenne semble être caractérisée par une faible densité de vestiges archéologiques d'époque gallo-romaine. Un urbanisme peu développé, l'absence de villa et la rareté des monuments épigraphiques sont autant d'indices qui paraissent désigner un territoire peu marqué par la conquête et la présence romaines. De récentes découvertes archéologiques viennent pourtant bouleverser cette vision historique simplifiée, en mettant au jour les traces d'activités minières et métallurgiques des premiers siècles de notre ère dans la vallée de Baigorri.

Initiée en 1999, l'étude de la métallurgie du fer a fait intervenir un certain nombre d'investigations complémentaires (étude bibliographique, enquête toponymique, prospection inventaire, études géophysiques et sondages archéologiques). Ces recherches ont dévoilé l'existence d'une production de fer au Pays Basque durant l'Antiquité, deux pôles d'activités minières et métallurgiques ayant été identifiés dans la vallée de Baigorri: les montagnes Larla et Errola ont livré les vestiges d'une production de fer qui semble être comprise entre le II siècle a.C. et le IVe siècle p.C. L'approche archéologique de l'activité antique s'insère dans un Programme Collectif de Recherche "Paléoenvironnement et dynamiques de l'anthropisation de la montagne basque", un dispositif interdisciplinaire orienté sur l'histoire de l'environnement. Les recherches paléoenvironnementales qui allient l'analyse palynologique et géochimique de tourbières, l'étude anthracologique de différents dépôts de charbon de bois (ateliers métallurgiques, charbonnières et mines) et l'étude dendrochronologique de bois de mines, visent à reconstituer l'histoire des activités minières et métallurgiques et les effets de celles-ci sur l'environnement de la vallée de Baigorri.

Il convient de présenter les premiers résultats de cette étude consacrée à l'histoire minière et métallurgique des Pyrénées occidentales, le premier bilan d'une démarche intégrant données archéologiques, historiques et paléoenvironnementales.

\section{LE PAYSAGE MINIER ET MÉTALLURGIQUE DE LA VALLÉE DE BAIGORRI}

\section{La diversité des gîtes métallifères}

D'un point de vue minéralogique, la vallée de Baigorri présente une certaine richesse par la quantité et, surtout, la variété des gîtes métallifères qu'elle recèle. Des minéralisations à sidérite (carbonate de fer, FeCO3) et chalcopyrite (sulfure de cuivre, $\mathrm{CuFeS2}$ ) se répartissent sur un large secteur compris entre le massif de l'Iparla au nordouest et l'extrémité de la vallée des Aldudes au sudest. Dans les terrains du Permo-Trias (posthercyniens) ces minéralisations sont constituées presque exclusivement de sidérite, le quartz et la chalcopyrite étant rares. Dans les séries paléozoïques, en revanche, la sidérite est associée à de la chalcopyrite et à des cuivres gris (sulfures de cuivre et d'argent) dans les terrains ordoviciens, ainsi qu'à des indices de cuivre, plomb et zinc dans les terrains du Dévonien. D'une manière générale, on peut observer un lien entre la lithologie des terrains et les minerais qu'ils contiennent: les niveaux détritiques de l'Ordovicien présentent des indices de cuivre et de fer, tandis que les séries du Dévonien contiennent du cuivre, du fer, du plomb et du zinc ${ }^{1}$.

\section{Le contexte archéologique. L'exploita- tion des ressources métallifères depuis la protohistoire}

La concentration et la diversité des minéralisations sur une aire géographique relativement restreinte constituent un important potentiel minier. Dès avant l'Antiquité et parfois jusqu'au Xxe siècle, les gisements métallifères du massif des Aldudes ont été exploités. Les traces d'activités minières et métallurgiques passées s'y trouvent encore en abondance (fig. 1). Désignentelles une exploitation continue et incessante des gîtes? Quels métaux étaient les plus convoités, à quelle époque?

1. Gapillou 1995, 51-54. 


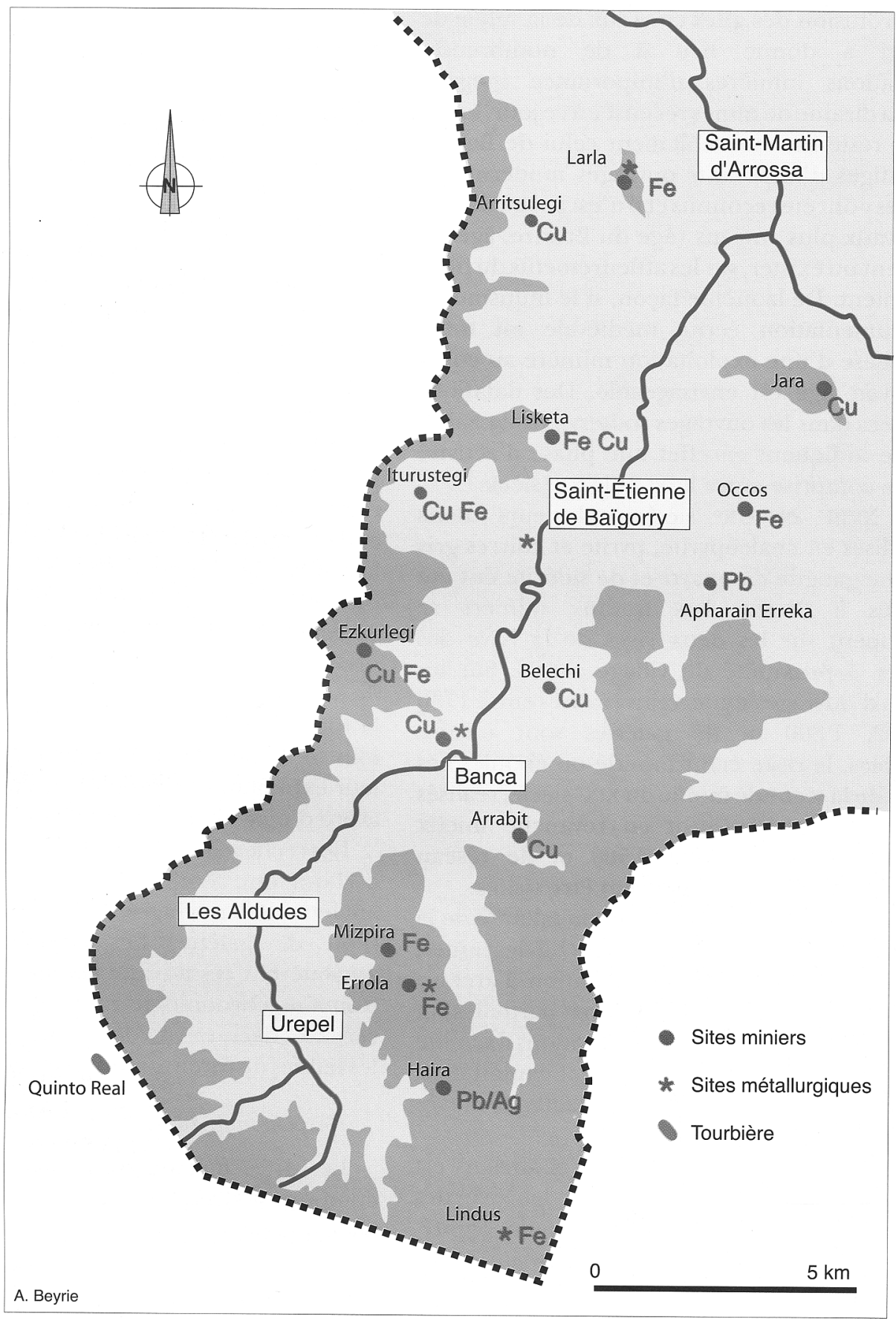

Fig. 1. Répartition des principaux sites miniers et métallurgiques en vallée de Baigorri. 


\section{L'exploitation du cuivre}

La profusion des gîtes cuivreux de la vallée de Baigorri a donné lieu à de nombreuses exploitations minières d'importance inégale. Parmi la dizaine de mines recensée à ce jour, le site majeur reste incontestablement celui de Banca. Les vestiges d'imposants ouvrages modernes et antiques y ont été reconnus et il n'est pas exclu que des travaux plus anciens (âge du Bronze, âge du Fer) aient pu exister, sur les affleurements du filon notamment. De la même façon, si le mutisme de la documentation écrite médiévale est total, l'hypothèse d'une exploitation minière au cours du Moyen Age est envisageable. Des datations effectuées dans les ouvrages souterrains de SaintAntoine indiquent en effet une phase d'activité minière comprise entre le $\mathrm{X}^{\mathrm{e}}$ et le $\mathrm{XII}^{\mathrm{e}}$ siècle ${ }^{2}$.

Aux XVIII et XIX $^{\mathrm{e}}$ siècles, plusieurs filons minéralisés en chalcopyrite, pyrite et cuivres gris dans une gangue de quartz et de sidérite ont été exploités à Banca. Les travaux miniers se développent sur les deux rives de la Nive des Aldudes, à proximité du village actuel. Sur les 6500 m d'ouvrages souterrains réalisés entre 1730 et $1782,1800 \mathrm{~m}$ de galeries sont encore accessibles, le reste étant inondé ou éboulé. Les travaux de la seconde moitié du XIX ${ }^{e}$ siècle, réalisés dans des quartzites, sont en revanche mieux conservés. C'est au total $3500 \mathrm{~m}$ de réseau souterrain moderne qui peuvent être visités.

L'évaluation de la production métallique de la fonderie, édifiée en 1747 au débouché des mines, reste approximative, la documentation d'archive ne livrant que des informations partielles et ponctuelles. D'après diverses estimations il semble néanmoins qu'au XvIII e siècle, l'exploitation aurait fourni dans son ensemble $1200 \mathrm{t}$ de cuivre métal et 12 à 15 t d'argent métal ${ }^{3}$.

La majorité des ouvrages miniers anciens est concentrée sur la rive gauche de la Nive des Aldudes, sur les filons de Berg-op-Zoom, SainteMarie et des Trois-Rois. La chalcopyrite et les

2. Il s'agit d'une datation par radiocarbone effectuée sur un charbon de bois prélevé dans les travaux souterrains de la mine

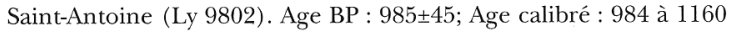
p.C. (Ancel 2000, 41).

3. Le filon des Trois-Rois aurait produit à lui seul 1000 t de cuivre et 10 t d'argent (Vié 1979, 13). cuivres gris y ont été exploités exclusivement pour le cuivre. A l'heure actuelle, environ $400 \mathrm{~m}$ de galeries anciennes ont été identifiées, sans compter les chambres d'exploitation. Les ouvrages de recherche, les imposants dépilages, les dispositifs d'exhaure et d'aérage dévoilent toute la technicité des mineurs et la rationalité de l'exploitation ${ }^{4}$. Si les mines de Banca paraissent évoluées sur le plan des techniques minières, la métallurgie ancienne demeure à ce jour totalement méconnue.

\section{Les mines de galène}

Le traitement des cuivres gris argentifères extraits des mines de cuivre de Banca au cours du XVIII ${ }^{\mathrm{e}}$ siècle exigeait d'importantes quantités de plomb (ce métal était nécessaire aux procédés métallurgiques permettant la séparation du cuivre et de l'argent). Les prospections entreprises dans la vallée de Baigorri révélèrent l'existence de plusieurs minéralisations plombifères susceptibles de donner un nouvel essor à la production métallique de la fonderie. Des indices de plomb avaient été décelés dans le vallon et dans la forêt d'Haira sur la commune d'Urepel, non loin de l'établissement métallurgique. En 1758, leur exploitation fut interrompue, le filon s'étant avéré d'épaisseur et de continuité variables.

Les recherches minières au XVIII ${ }^{\mathrm{e}}$ siècle en forêt d'Haira ont révélé l'existence de travaux plus anciens. Des ouvrages avaient été réalisés sur le filon de galène. Les exploitants modernes attribuèrent ces travaux éboulés et enfoncés aux Romains, chronologie que viennent confirmer les récentes découvertes de mobilier céramique dans les haldes de travaux miniers de la forêt de Haira ${ }^{5}$.

\section{L'exploitation du fer}

Les gîtes ferreux, principalement des filons comportant de la sidérite, abondent dans la vallée au sein de divers faciès géologiques (Ordovicien,

4. L'essentiel des recherches archéologiques menées sur le réseau minier antique de Banca concerne l'exploitation du filon des TroisRois (Ancel et al. 2001, 119-139).

5. Des fragments de lampe à huile et de céramique sigillée du $I^{\text {er }}$ siècle p.C. (lampe à volute et sigillée gallo-romaine de forme Drag. 25 notamment) gisaient à la surface de déblais de mines souterraines et de travaux à ciel ouvert. 
Dévonien et Permo-Trias). Ces minéralisations ont fait l'objet d'exploitations successives depuis la fin des périodes protohistoriques jusqu'au $\mathrm{XX}^{\mathrm{e}}$ siècle.

L'existence d'un district minier et métallurgique antérieur à l'époque gallo-romaine est en effet attestée dans la haute vallée, sur la montagne Errola (Urepel). Des vestiges de bas fourneaux appartenant à des ateliers métallurgiques du $\mathrm{II}^{\mathrm{e}}$ siècle a.C. y ont été mis au jour, à proximité de mines à ciel ouvert. Si de nombreux vestiges similaires jalonnent l'ensemble de la vallée, c'est sans aucun doute sur le massif de Larla (Saint-Étienne-de-Baigorry / Saint-Martin-d'Arrossa) que l'activité sidérurgique antique fut la plus dense. Les vestiges d'une trentaine d'ateliers métallurgiques y ont été identifiés, jouxtant d'imposants travaux réalisés sur un filon de sidérite.

Convoité dès les premiers siècles de notre ère, ce puissant filon dit d'Ustelegi, fut activement exploité à l'époque moderne. Principal gîte ferreux de la vallée, il en alimenta successivement les divers établissements métallurgiques. A la fin du XVIII siècle, Palassou, puis Diétrich, précisent en effet que la forge du vicomte d'Etxauz, fondée en 1640 en amont du village de Saint-Étienne-deBaïgorry, avait été alimentée par de la mine de fer spathique, Minera ferri alba spathifurmis, tirée au lieu d'Usteley, au nord du château d'Etxauz. L'exploitation minière perdura vraisemblablement jusqu'en 1785, date à laquelle la forge cessa toute activité faute de combustible. Au tout début du XIXe siècle, la construction d'une forge à la catalane à Banca, sur les ruines de la fonderie de cuivre, relança l'exploitation du filon. L'établissement édifié en 1800 fonctionna quatre ans et fut alimenté par la sidérite du gisement voisin. Toujours dans le premier quart du XIXe siècle, la richesse du filon d'Ustelegi suscita la construction d'un haut fourneau à Banca. Dès 1823, les travaux de construction de l'usine à fer commencèrent, tandis qu'en 1826 le haut fourneau fut mis en service. Il connaîtra une activité discontinue jusqu'en 1850.

Des études menées à la fin du XIXe siècle révélèrent toute l'importance du gîte, ainsi que son fort potentiel économique. Il semblait que la fracture à remplissage sidéritique qui se développait dans les grès du Permo-Trias se poursuivait en profondeur, dans les schistes de l'Ordovicien. L'exploitation fut relancée dès 1906, mais les niveaux schisteux s'avérèrent bien vite stériles et, le 3 juillet 1914, la société des mines d'Ossès et de Banca fut dissoute. Le filon d'Ustelegi était épuisé.

\section{L'histoire environnementale de la vallée. Les apports de la palynologie et de la géochimie isotopique du plomb}

Une démarche paléoenvironnementale intégrée a été engagée dans la haute vallée de Baigorri afin d'appréhender sur la longue durée à la fois l'histoire des activités métallurgiques et leurs conséquences environnementales ${ }^{6}$. La séquence de Quinto Réal, prélevée dans une tourbière située à $910 \mathrm{~m}$ d'altitude sur le massif séparant la vallée du Baztan de celle des Aldudes (fig. 1), a fait l'objet d'une étude croisée combinant des analyses palynologiques classiques avec une analyse de géochimie isotopique du plomb. Cette méthode, qui s'appuie sur "la mémoire du plomb", permet de mettre en évidence les phases de paléopollutions liées aux activités métallurgiques ou extractives.

L'étude géochimique des archives naturelles telles que les tourbières ${ }^{7}$ permet de retracer l'histoire des dépôts atmosphériques en micropolluants métalliques, et donc de reconstruire en partie la chronologie des grandes phases d'exploitation. Deux problèmes se posent toutefois. Ils concernent d'une part la bonne conservation du signal géochimique contenu dans l'archive, et d'autre part les capacités de discrimination des apports locaux face à la contribution continentale. En outre, la seule mesure des concentrations en métaux lourds peut engendrer certaines ambiguïtés, notamment dans les cas où les variations observées résultent de phénomènes naturels comme par exemple l'augmentation des apports détritiques résultant

\footnotetext{
6. Galop et al. 2001 ; Galop et al. (sous presse) ; Monna et al. (à paraître).

7. Shotyk et al. 1996 ; Martinez-Cortizas et al. 1997; Weiss et al. 1997 ; Görres \& Frenzel 1997 ; Kempter et al. 1997 ; Mackenzie et al. 1998 ; Kempter \& Frenzel 1999, 2000 ; Shotyk et al. 2000.
} 
Quinto réal - 910 m. (Baigorri valley; Navarra - Spain)

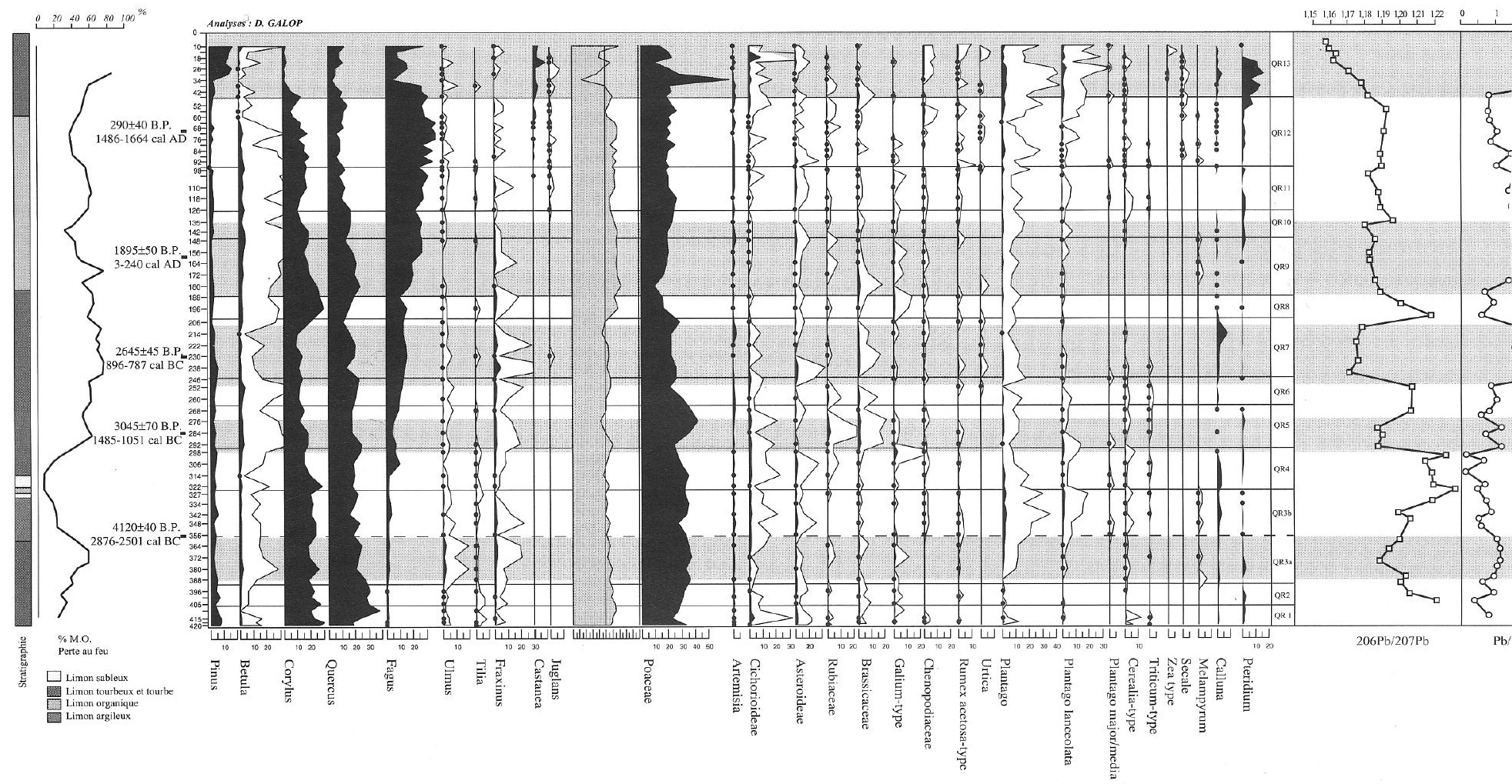

Fig. 2. Diagramme pollinique simplifié de Quinto Real.

d'une déforestation massive. C'est pour cette raison que dans ce domaine l'utilisation des techniques isotopiques du plomb s'est développée depuis peu ${ }^{8}$. En effet le plomb possède des caractéristiques uniques qui le rendent particulièrement bien adapté à cette recherche ${ }^{9}$. Il est composé de quatre isotopes stables : ${ }^{204} \mathrm{~Pb}$, ${ }^{206} \mathrm{~Pb},{ }^{207} \mathrm{~Pb}$ et ${ }^{208} \mathrm{~Pb}$. Le premier de ces isotopes $\left({ }^{204} \mathrm{~Pb}\right)$ n'est pas radiogénique, c'est-à-dire qu'il n'est pas issu de la désintégration d'un isotope radioactif. Son abondance est donc restée identique depuis la formation de la Terre. Les trois autres isotopes $\left({ }^{206} \mathrm{~Pb},{ }^{207} \mathrm{~Pb}\right.$ et $\left.{ }^{208} \mathrm{~Pb}\right)$ sont produits de façon continue au cours du temps par la désintégration d'isotopes radioactifs : ${ }^{238} \mathrm{U},{ }^{235} \mathrm{U}$ et ${ }^{232} \mathrm{Th}$. Pour simplifier, lors de la ségrégation d'une minéralisation, en général sulfurée comme la

8. Brännvall et al. 1997; Rosman et al. 1997; Shotyk et al. 1998; Dunlap et al. 1999 ; Bindler et al. 1999 ; Renberg et al. 2000 ; Monna et al. 2000.

9. Faure 1986 ; Kramers \& Tolstikhin 1997. galène, le plomb est isolé de ses isotopes pères ( $\mathrm{U}$ et $\mathrm{Th}$ ); sa composition isotopique s'en trouve "gelée"; elle n'évolue plus à partir de la cristallisation. A l'opposé, dans les roches et les sols, la production en plomb radiogénique continue en présence d'isotopes pères. Bien que cela ne soit pas toujours vrai, les minéralisations sont souvent moins radiogéniques que les terrains encaissants. Il est donc possible de distinguer un plomb issu de l'érosion du substratum de celui qui a été remobilisé par l'homme dans l'environnement à la suite des pratiques minières ou métallurgiques. Les aspects méthodologiques de la technique utilisée étant décrits par ailleurs ${ }^{10}$, nous retiendrons dans le cadre de cet article que deux outils géochimiques principaux ont été mobilisés pour caractériser les apports de micropolluants d'origine anthropique: les

10. Galop et al. 2001 ; Galop et al. (sous presse) ; Monna et al. (à paraître). 
rapport $\mathrm{Pb} / \mathrm{Sc}$ et ${ }^{206} \mathrm{~Pb} /{ }^{207} \mathrm{~Pb}$, en précisant qu'une augmentation des rapports $\mathrm{Pb} / \mathrm{Sc}$ associée à une baisse simultanée des rapports ${ }^{206} \mathrm{~Pb} /{ }^{207} \mathrm{~Pb}$ atteste une intensification des apports atmosphériques de micro-polluant d'origine anthropique. Les courbes de ces rapports sont portées à droite du diagramme pollinique synthétique (fig. 2).

L'examen des courbes isotopiques (fig. 2) révèle l'existence de quatre phases de pollutions objectivement reliables à des périodes d'intensification des activités métallurgiques ou minières. Il s'agit de l'âge du Bronze moyen médocain (zone QR5 ; XVe-XVI ${ }^{\mathrm{e}}$ siècles a.C.) ; de l'âge du Bronze final (zone QR 7 ; IXe siècle a.C.), de la période couvrant la fin de l'âge du Fer et l'Antiquité (zones QR9 et QR 10 ; II siècle a.C.- IV siècle p.C.) et enfin de l'époque moderne à partir du XVIII siècle. Toutefois, si ces phases de pollution correspondent à des épisodes reconnus dans l'histoire de la métallurgie régionale, il convient de souligner également l'existence d'un signal géochimique plus ancien, antérieur à $4120 \pm 40$ BP (2876-2501 cal BC). Les variations des rapports isotopiques enregistrées dans la zone QR3a, pourraient être la conséquence d'activités extractives et/ou métallurgiques aux environs de 3200-3000 a.C. C'est d'ailleurs précisément durant cette période que semble s'opérer un décollage des productions métalliques en France du sud ${ }^{11}$. Cependant, bien que l'hypothèse d'une métallurgie ancienne ait été évoquée ${ }^{12}$, des doutes persistent et nous empêchent de conclure. Ainsi, associé au fait qu'il n'existe localement encore aucune trace d'une métallurgie aussi ancienne, il est fort probable que l'existence dans la séquence sédimentaire d'un horizon détritique situé vers $320 \mathrm{~cm}$ a pu faciliter la migration du plomb vers des horizons sous-jacents. Pour l'heure, cette piste de recherche reste ouverte et des études complémentaires sont en cours dans d'autres sites sédimentaires.

Ces pollutions semblent témoigner d'activités locales ou micro-régionales (massif ou vallée) ; c'est du moins ce que semblent indiquer les données polliniques. Durant la période de l'âge

11. Guilaine 1997 ; Ambert 1997.

12. Galop et al. 2001. du Bronze (QR5), la diminution des valeurs du chêne, du noisetier et, dans une moindre mesure, du hêtre (Fagus) est associée à une élévation des fréquences polliniques du bouleau et des Poacées. De même, durant la phase $Q R 7$, des signaux de déforestation sont perceptibles par un recul du chêne, du bouleau ainsi que par la hausse de certaines héliophiles, telle que la callune (Calluna). Dans les deux cas, et cela semble paradoxal, les indices polliniques d'activités agropastorales (Plantago lanceolata, Plantago major/ media, Melampyrum, Rumex) sont faiblement représentés ou en régression par rapport aux périodes précédentes. Cette caractéristique est nette dans la zone QR7, dans laquelle les indices polliniques d'agriculture disparaissent, à l'exception des orties (Urtica) et des plantains qui témoignent du maintien d'une fréquentation pastorale. Il ressort de ces résultats que des déboisements, quoique modérés, ont été réalisés aux environs du site dans des contextes de faible pression, voire de déprise agro-pastorale. Par conséquent, il paraît raisonnable d'envisager d'autres causes pour expliquer cette réduction de la forêt et l'éventualité de prélèvements destinés aux activités métallurgiques peut être formulée. D'autres éléments sont à verser au crédit de cette hypothèse, en particulier les indices de reforestation enregistrés entre ces deux périodes d'activités, lors des phases QR6 et QR8. Ces phases correspondent systématiquement à une baisse du rapport $\mathrm{Pb} / \mathrm{Sc}$ et à une augmentation du rapport ${ }^{206} \mathrm{~Pb} /{ }^{207} \mathrm{~Pb}$ qui signalent un affaiblissement des activités extractives et/ou métallurgiques. Un lien direct entre activités minières/métallurgiques et dynamique de la couverture forestière locale semble donc exister, ce qui renforce l'éventualité d'activités métallurgiques ou extractives localisées dans cette partie de la haute vallée de Baigorri.

Postérieurement, pour la période antique, les données palynologiques indiquent une reprise des déforestations, qui affectent le chêne et le noisetier, tandis que le hêtre semble au contraire s'étendre. L'ouverture du milieu est confirmée par une légère augmentation des Poacées, par l'extension du bouleau, ainsi que par la présence de Melampyrum, qui pourrait indiquer l'existence de faciès incendiés. Là encore, comme pour les phases antérieures, les signes d'une réduction de 
la forêt apparaissent sans qu'aucun indice ne traduise la présence d'activités culturales (QR9). Seules les faibles représentations de Plantago lanceolata attestent une anthropisation modérée du milieu. Toutefois, l'impact de ces activités sur les forêts reste vraisemblablement encore modeste. Il concerne apparemment le chêne et les formations secondaires de moyenne altitude (vallée et bas de versants ?) qui lui sont associées. A l'exception des données polliniques, nous ne disposons encore que de peu d'informations paléobotaniques. Toutefois, les premières analyses anthracologiques réalisées dans les crassiers des bas fourneaux d'Errola montrent une très nette prédominance de charbon de chêne ${ }^{13}$, tandis que la majorité des bois extraits des galeries antiques de la mine de Banca est également identifiée comme appartenant à la même essence. Quoique encore mal assurée, l'hypothèse d'un prélèvement sélectif pourrait être avancée.

C'est au cours de la période moderne que l'on observe une rupture dans l'histoire environnementale. La représentation pollinique de l'ensemble des essences forestières (Fagus, Quercus, Corylus) s'effondre et reflète un déboisement important des environs de la tourbière. Cette ouverture de l'espace forestier est également signalée par une hausse des herbacées héliophiles ainsi que par une extension des landes. Simultanément, le rapport $\mathrm{Pb} / \mathrm{Sc}$ grimpe en flèche, tandis que le rapport isotopique ${ }^{206} \mathrm{~Pb} /$ ${ }^{20} \mathrm{~Pb}$ chute brutalement. Il est certain que l'essentiel du signal anthropique est local, car les quantités de métal impliquées sont trop importantes pour être le résultat d'un apport atmosphérique de large amplitude. Nous retrouvons dans cette anomalie le décollage de l'activité métallurgique qui caractérise la vallée de Baigorri, et plus largement le Pays Basque, à la fin du Moyen Age ${ }^{14}$. Nos estimations chronologiques permettent de situer ce démarrage vers 1750 , date à laquelle commence à fonctionner la fonderie de cuivre de Banca (1747). La consommation en charbon de bois nécessaire au fonctionnement de ces établissements était considérable et l'essentiel

13. Mougin 2001

14. Goyehenetxe 2001. de l'espace forestier de la vallée était dévolu au charbonnage, tout comme une grande partie des forêts du Pays Basque ${ }^{15}$. Le synchronisme entre la déforestation enregistrée par les données pollenanalytiques et cette intensification de la pression métallurgique n'a rien de surprenant, d'autant plus que les vestiges de charbonnières repérés lors des prospections dans les forêts voisines du site traduisent l'intensité des prélèvements destinés à l'alimentation des forges de la vallée. Par ailleurs, les données textuelles indiquent que durant le XvIII ${ }^{e}$ siècle les forêts du Pays Quint avaient été détruites en totalité pour l'alimentation des forges ${ }^{16}$, ce qui paraît exact lorsque l'on considère la chute drastique du hêtre enregistrée durant cette période.

\section{APPROCHE ARCHÉOLOGIQUE DE L'EXPLOITATION ANTIQUE DU FER}

L'étude du paysage minier et métallurgique de l'extrémité occidentale de la chaîne pyrénéenne, ou plus exactement celle de la vallée de Baigorri, a mis en évidence plusieurs phases d'exploitation et de production métallique comprises entre l'âge du Bronze moyen médocain (XVe-XIVe siècle a.C.) et le $\mathrm{XX}^{\mathrm{e}}$ siècle. Le redémarrage des activités métallurgiques à l'époque antique, révélé par les résultats des analyses palynologiques et géochimiques de la tourbière de Quinto Réal, semble être confirmé par les évidences archéologiques. A l'heure actuelle, en effet, pas moins de quatre districts miniers et/ou métallurgiques antiques sont avérés en vallée de Baigorri. Dans un tel contexte, les indices liés au fer, manifestement les plus abondants et paradoxalement les moins étudiés, méritent un éclairage original.

\section{Méthodes d'investigation}

Les travaux entrepris sur les centres antiques de production de fer ont suivi une démarche méthodologique dont les trois temps forts retracent chronologiquement quatre années de recherche et de travaux de terrain. La première

15. Lefebvre 1933.

16. Hourmat 1995. 
phase de l'étude devait permettre de recenser les divers ateliers métallurgiques, estimer leur importance et observer leur répartition, mais aussi reconnaître les mines et les charbonnières susceptibles d'avoir alimenté les bas fourneaux de réduction. Plus concrètement, cette étude, qui visait à saisir l'organisation et l'amplitude des activités minières et métallurgiques au sein de chacun des districts, a consisté en un conséquent travail de terrain incluant pour l'essentiel prospections systématiques, levés topographiques et reports cartographiques. Parallèlement à ces investigations, une phase d'évaluation chronologique était entreprise par le biais de sondages archéologiques. Ces derniers, conduits sur onze des quarante ferriers découverts dans les massifs de Larla et d'Errola, devaient révéler une activité métallurgique comprise entre la fin de l'âge du Fer et le Bas Empire (II siècle a.C.-III ${ }^{\mathrm{e}}$ siècle p.C.). Au terme de ces recherches préliminaires, des investigations archéologiques ont porté sur quatre ateliers distincts. Il s'agissait de confirmer la chronologie des activités, mais surtout de mettre au jour les bas fourneaux de réduction, d'en étudier les structures et d'en observer les caractéristiques techniques. Les fouilles devaient en outre permettre de saisir l'organisation spatiale des ateliers.

\section{Une production métallique au $\mathrm{II}^{\mathrm{e}}$ siècle a.C. dans la haute vallée de Baigorri. La montagne Errola}

\section{De l'extraction à la production métallique}

— Contexte géologique et type d'extraction

Les travaux miniers de la montagne Errola ont été entrepris dans des terrains primaires composés de quartzites, de schistes et de formations calcareo-dolomitiques de l'Ordovicien et du Dévonien. Aucun indice de minéralisation n'est signalé dans la bibliographie, bien que d'imposants ouvrages miniers à ciel ouvert aient été réalisés sur des affleurements filoniens ${ }^{17}$. On distingue deux grands types de sites d'extraction : une exploitation en fosses grossièrement circulaires (Meharroztegi 2) et des travaux associant fosses et tranchées oblongues (Errola 4) ${ }^{18}$ (fig. 3).

\section{— Les indices métallurgiques}

Sept ferriers ont été recensés dans les montagnes d'Errola et d'Otsamunho. Ces indicateurs d'activité métallurgique sont répartis sur le versant ouest du massif, à une altitude comprise entre 665 et $780 \mathrm{~m}$, sur une longueur de 2,5 km. Les sites sont généralement proches des secteurs miniers. C'est le cas des ateliers Meharroztegi 1 et 3, qui jouxtent les fosses de Meharroztegi 2, mais aussi des sites Errola 3, 5, et 6 qui furent installés soit au sommet, soit directement en aval des tranchées d'extraction Errola 4. L'alimentation d'un atelier, l'atelier Errola 1, reste problématique, puisque jusqu'à présent aucun travail minier n'a été identifié à proximité du site de réduction.

\section{— Les zones de charbonnage}

On connaît l'existence de plusieurs charbonnières sur les éminences d'Errola et d'Otsamunho. L'une d'elles se situe environ à 250 $\mathrm{m}$ au sud/sud-est de l'atelier Errola 3, tandis qu'une des trois charbonnières repérées sur Otsamunho est implantée à moins de 100 m en amont de Errola 1.

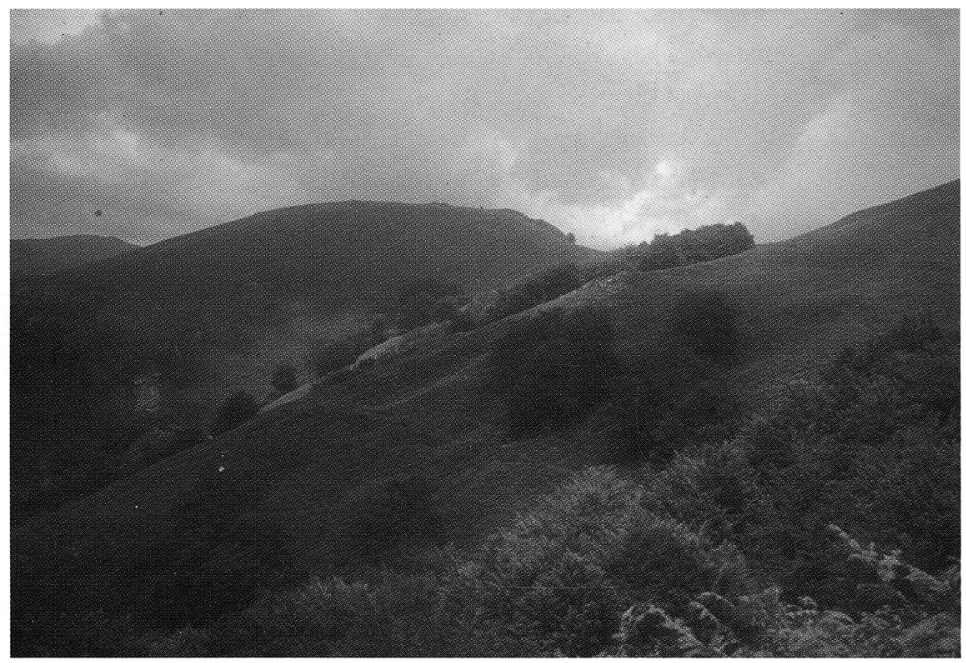

Fig. 3. Mines à ciel ouvert de la montagne Errola (Errola 4).

17. L'examen des haldes semble indiquer que les ouvrages miniers ont exploité les parties supérieures d'un filon de sidérite. On note également la présence de goethite aux abords des fosses d'extraction (chapeau de fer).

18. L'étude topographique de ces vestiges est en cours de réalisation. 
Le lien entre cette production de charbon et l'activité métallurgique ancienne n'étant pas attesté, des études anthracologiques ont été entreprises de façon conjointe sur des charbonnières et sur des ateliers de réduction. L'analyse des charbons de bois prélevés dans trois charbonnières a révélé une utilisation exclusive de bois de hêtre, tandis que l'étude des charbons de bois présents dans les déchets métallurgiques antiques des sites Errola 3 et Errola 5 a montré une utilisation majoritaire de bois de chêne et de châtaigner, le hêtre ne constituant que $4 \%$ des fragments prélevés sur le site Errola $5{ }^{19}$. Il apparaît clairement que le charbon de bois produit dans les charbonnières analysées n'a pas servi à la production métallique ancienne du massif. Postérieures aux activités métallurgiques, les zones de charbonnage trahissent une exploitation moderne de la hêtraie.

\section{Les ateliers métallurgiques Errola 3 et Errola 5}

\section{- Errola 3}

Le site Errola 3 fait partie des six sites métallurgiques découverts au cours des prospections réalisées en 2000. La datation des charbons de bois prélevés dans le crassier a révélé une période d'activité ancienne comprise entre 148 a.C. et 48 p.C. 20

L'atelier se présente sous la forme d'une petite plate-forme artificielle (moins de $200 \quad \mathrm{~m}^{2}$ ) aménagée à flanc de montagne (fig. 4), directement en amont des mines à ciel ouvert Errola 4. Un sondage archéologique (S.1.: 1 x $1,90 \mathrm{~m}$ ), réalisé à la limite occidentale de la plate-forme en rupture de pente, a mis au jour une couche de déchets métallurgiques relativement dense, mais peu épaisse $(0,15$ à $0,30 \mathrm{~m})$ et peu étendue à en juger par l'épandage de scories visible en surface (long. max.: $15 \mathrm{~m}$ ). Ces données, corrélées à la topographie générale du site, semblaient indiquer que les déchets produits par un ou plusieurs fours de réduction étaient évacués depuis la terrasse artificielle vers l'aval, sur

19. Errola 3: Crataegus $25 \%$; Quercus fc $25 \%$; Ribes $50 \%$. Errola 5 : Castanea sativa/Quercus fc $12 \%$; Fagus sylvatica $4 \%$; Quercus fc $84 \%$ (Mougin 2001, 59-61).

20. LY-10658. Age B.P. $2040 \pm 30$. Pics de probabilité -5, -25, -43, -85. les pentes naturelles du site, afin d'éviter tout encombrement de l'aire de travail. Il paraissait vraisemblable que cette surface plane ait pu abriter les bas fourneaux de réduction de l'atelier.

La prospection magnétique ${ }^{21}$ a sensiblement bouleversé cette perception de l'organisation spatiale de l'atelier métallurgique antique. Quatre ensembles de fortes valeurs de susceptibilités magnétiques ont été mis en évidence, tous situés en marge de la plate-forme. Ainsi, contre toute attente, aucune anomalie susceptible de désigner l'emplacement d'un four de réduction n'a été décelée sur la partie plane et centrale du site. L'anomalie majeure signalait la localisation possible d'un bas fourneau dans la partie sommitale de la butte qui borde la plate-forme au nord. Deux autres anomalies marquaient deux emplacements éventuels de four, sur le petit plateau qui surplombe la plate-forme au sud cette fois.

Un sondage (S.2. : 3,5 $\mathrm{m}^{2}$ ) a été implanté sur l'emplacement hypothétique d'un bas fourneau. Si les vestiges d'un four de réduction n'ont pas été formellement identifiés, l'ensemble des aménagements et des niveaux archéologiques mis au jour à l'intérieur du sondage désigne bien un atelier métallurgique. Une fosse creusée dans le substrat rocheux (schiste) présente les caractéristiques des fosses aménagées à l'avant des bas fourneaux de réduction. Elle semble avoir été conçue pour permettre l'écoulement des scories, faciliter les défournements et réaliser les diverses opérations de maintenance du four telles que la réfection du revêtement interne de la cuve ou encore la reconstruction de la porte. Les divers éléments qui constituent son remplissage (amas de nodules d'argile réfractaire plus ou moins cuits, fragments de paroi de four, volumineuses scories coulées et charbons de bois) témoignent de ces multiples activités. Directement au sud de cet aménagement, un amoncellement de blocs d'argile réfractaire cuits et scorifiés résulte de l'effondrement ou de la destruction d'une structure de réduction. Ces éléments

21. L'étude géophysique de quatre ateliers métallurgiques de la vallée de Baigorri a été entreprise au mois de juin 2001 par I. Guyot. Elle concerne les sites Errola 3, Errola 5, Harotzainekoborda et Haritzondo. (Guyot 2001, 3-4). 


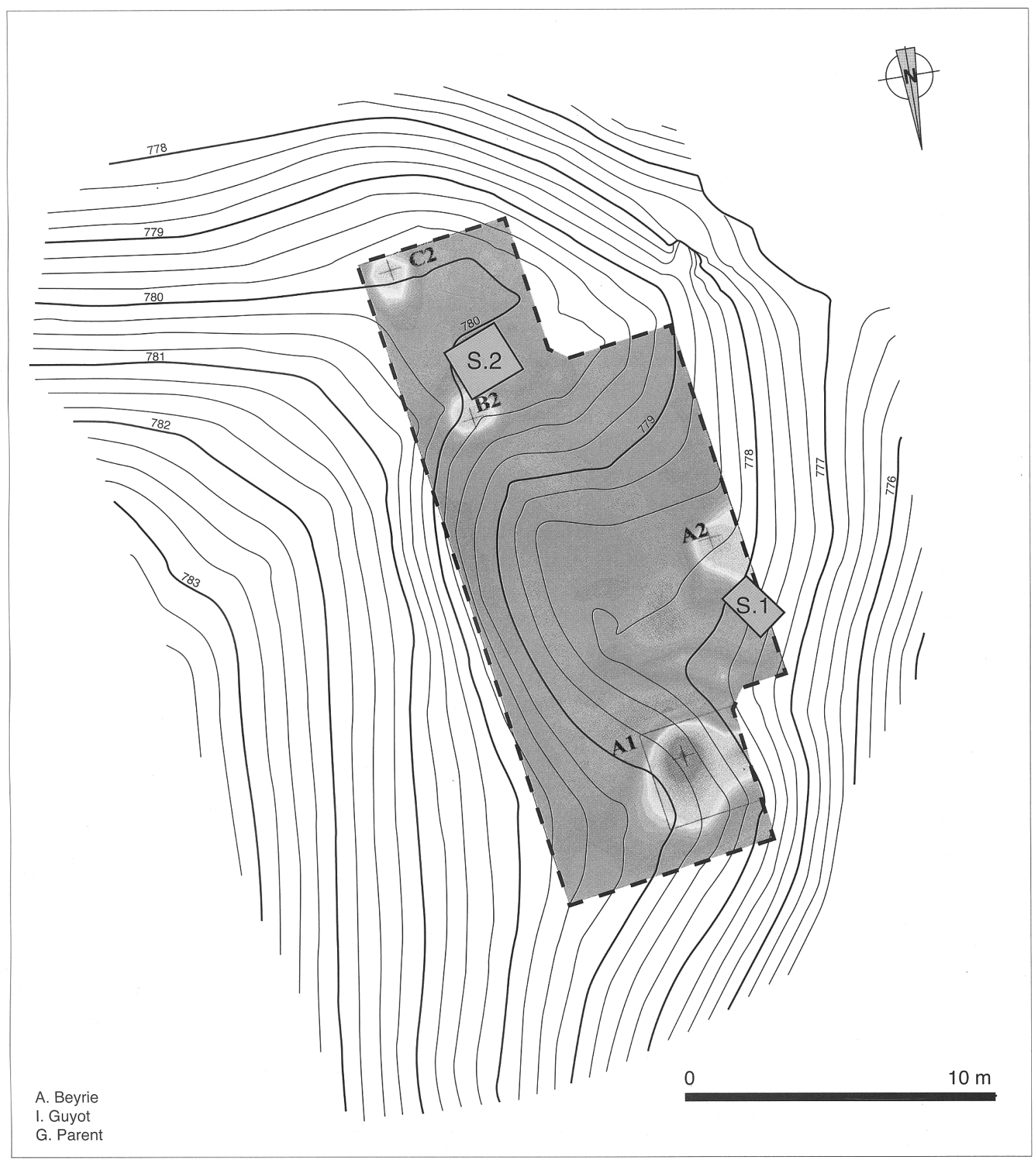

Fig. 4. Topographie et carte magnétique de l'atelier métallurgique Errola 3. 
appartenaient certainement à la cuve d'un bas fourneau.

Des installations métallurgiques occupaient donc la partie sommitale de la butte qui surplombe au sud la plate-forme centrale d'Errola 3, tandis que la carte géophysique du site suggère l'existence d'une seconde structure de réduction sur la butte opposée, au nord de l'esplanade. Les déchets métallurgiques produits étaient évacués en aval de la plate-forme, vers l'est, sur la pente naturelle.

Si l'on admet un tel schéma de gestion de l'espace de l'atelier Errola 3, c'est-à-dire une concentration des infrastructures métallurgiques en marge et en amont de la plate-forme centrale et une évacuation des déchets depuis ces pôles vers l'aval de la plate-forme, il reste encore à définir de quelle manière l'espace plan central était utilisé. Cette esplanade, qui ne semble intervenir ni dans les processus strictement métallurgiques, ni dans la gestion des déchets, était probablement utilisée pour des opérations annexes. Elle a pu servir à la préparation du minerai et à son stockage, de même qu'à l'entrepôt du charbon de bois nécessaire aux réductions. La volonté d'installer les fours de réduction sur des buttes ventées plutôt que sur la plate-forme centrale soulève la question du mode de fonctionnement des bas fourneaux d'Errola 3. Ces derniers requéraient-ils une ventilation naturelle ou forcée? Dans un cas comme dans l'autre, une telle exposition au vent devait faciliter la dispersion des émanations gazeuses produites par les bas fourneaux.

La découverte de clous en fer et de tessons de céramique fait preuve qu'une construction légère, de type appentis ou cabane, était aménagée sur la butte sud de l'atelier, non loin des fours de réduction.

\section{- Errola 5}

L'atelier Errola 5 se présente sous la forme d'une terrasse artificielle à la topographie beaucoup moins marquée qu'Errola 3. Il s'agit d'un léger replat aménagé sur la pente et en aval duquel un amoncellement de déchets métallurgiques s'est constitué. L'atelier était installé au pied des travaux miniers superficiels Errola 4, soit presque à $250 \mathrm{~m}$ au nord et en aval du site métallurgique Errola 3.
Un sondage (S.1. : 1 × 1,80 m) a permis de déterminer une phase chronologique de l'activité du site comprise entre 197 et 4 a.C. ${ }^{22}$, datation haute qui coïncidait avec la période d'activité d'Errola 3. Implanté en bordure de la plate-forme artificielle, le sondage a en outre mis au jour un aménagement (rigole ou fossé) pour l'évacuation, vers la pente, des divers déchets métallurgiques produits sur la plate-forme. Une tuyère gisait au fond de la rigole, parmi des scories et des fragments de paroi de four. Ces divers éléments laissaient augurer la présence d'un four de réduction en amont, sur la terrasse artificielle.

La prospection magnétique réalisée sur cette dernière et à ses abords immédiats a révélé deux anomalies pouvant indiquer la présence de bas fourneaux. L'une d'elles, celle qui se situait directement sur la plate-forme, a fait l'objet d'une vérification archéologique (S.2.: $\left.8 \mathrm{~m}^{2}\right)$. Les blocs de schiste et de plaques d'argile réfractaire amassés, mis au jour au centre du sondage, appartenaient selon toute vraisemblance à une structure de réduction effondrée (ERROLA.F.1.). Si l'on considère la nature et les dimensions des matériaux éboulés, il semble que le bas fourneau devait être de construction massive. Les éléments dont nous disposons à l'heure actuelle ne permettent malheureusement pas de restituer la morphologie et le mode opératoire de cette structure de réduction. La découverte d'une tuyère sur l'atelier (S.1.) permet tout de même d'envisager l'emploi d'une ventilation forcée à l'aide de soufflets.

\section{L'activité minière et métallurgique du massif de Larla}

\section{La répartition spatiale des activités antiques}

— Les ouvrages miniers

Le contexte minier du massif de Larla est très complexe, des travaux ayant été conduits au cours des deux derniers millénaires sur les diverses minéralisations de sidérite qui sillonnent la montagne. Si l'on a déjà évoqué les exploitations

22. LY-10659. Age B.P. $2095 \pm 30$. Pics de probabilité $-65,-95,-107$, -145 . 
modernes qui aboutirent à l'épuisement du gîte principal au début du $\mathrm{xx}^{\mathrm{e}}$ siècle, la découverte d'une trentaine d'ateliers métallurgiques, dont cinq assurément anciens, sur les flancs même de Larla, assure la mise en ouvre d'investigations minières assidues dès les premiers siècles de notre

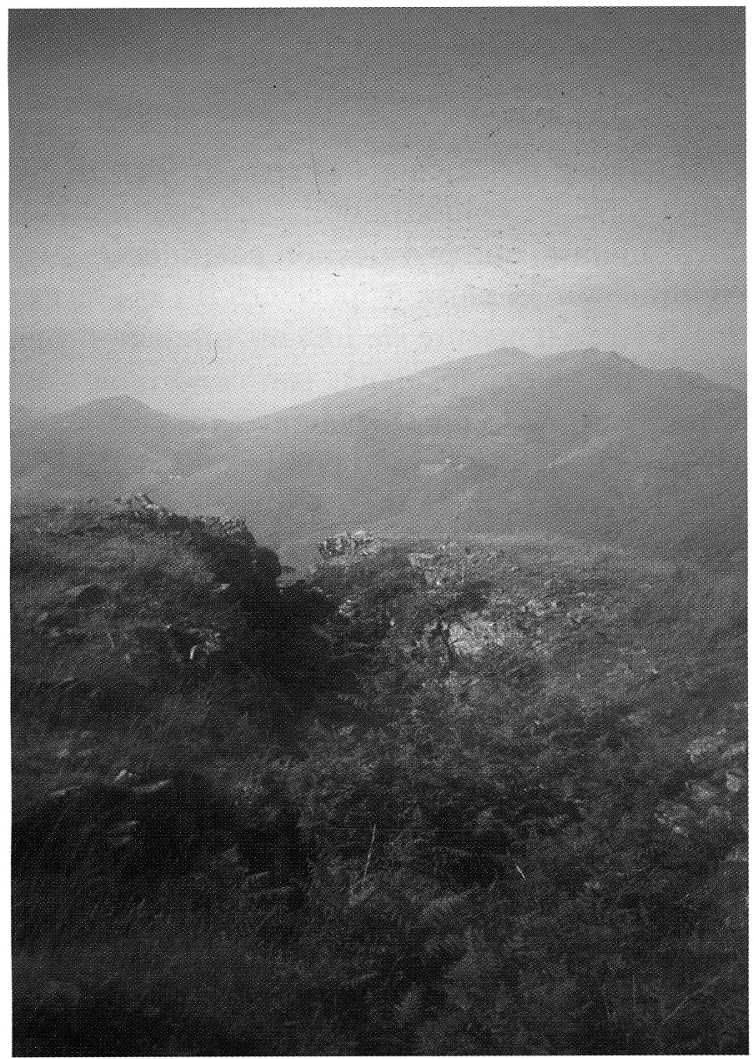

Fig. 5. Tranchée d'exploitation minière de la montagne Larla.

ère. L'activité minière la plus ancienne du massif, en l'occurrence antique, dut être vouée à l'exploitation des affleurements de sidérite et à celle du chapeau de fer (goethite). A ce stade de la recherche, même si la chronologie des ouvrages miniers n'est pas encore formellement établie, nous avons l'assurance qu'une partie des chantiers superficiels et souterrains qui s'étalent sur plus de $2 \mathrm{~km}$ de longueur (fig. 5) fournissait le minerai de fer nécessaire aux opérations de réduction directe pratiquées dans les ateliers métallurgiques établis sur la montagne de Larla.
— Les sites métallurgiques

Le nombre de ferriers recensés sur le massif s'élève à trente-trois. Les sites métallurgiques sont scindés en trois ensembles géographiques distincts : le groupe méridional composé de dixsept crassiers concentrés sur une zone de 75 ha ; l'ensemble septentrional comprenant dix ateliers répartis sur près de 70 ha; le groupe aval qui compte six ferriers (fig. 6). Cette délocalisation de

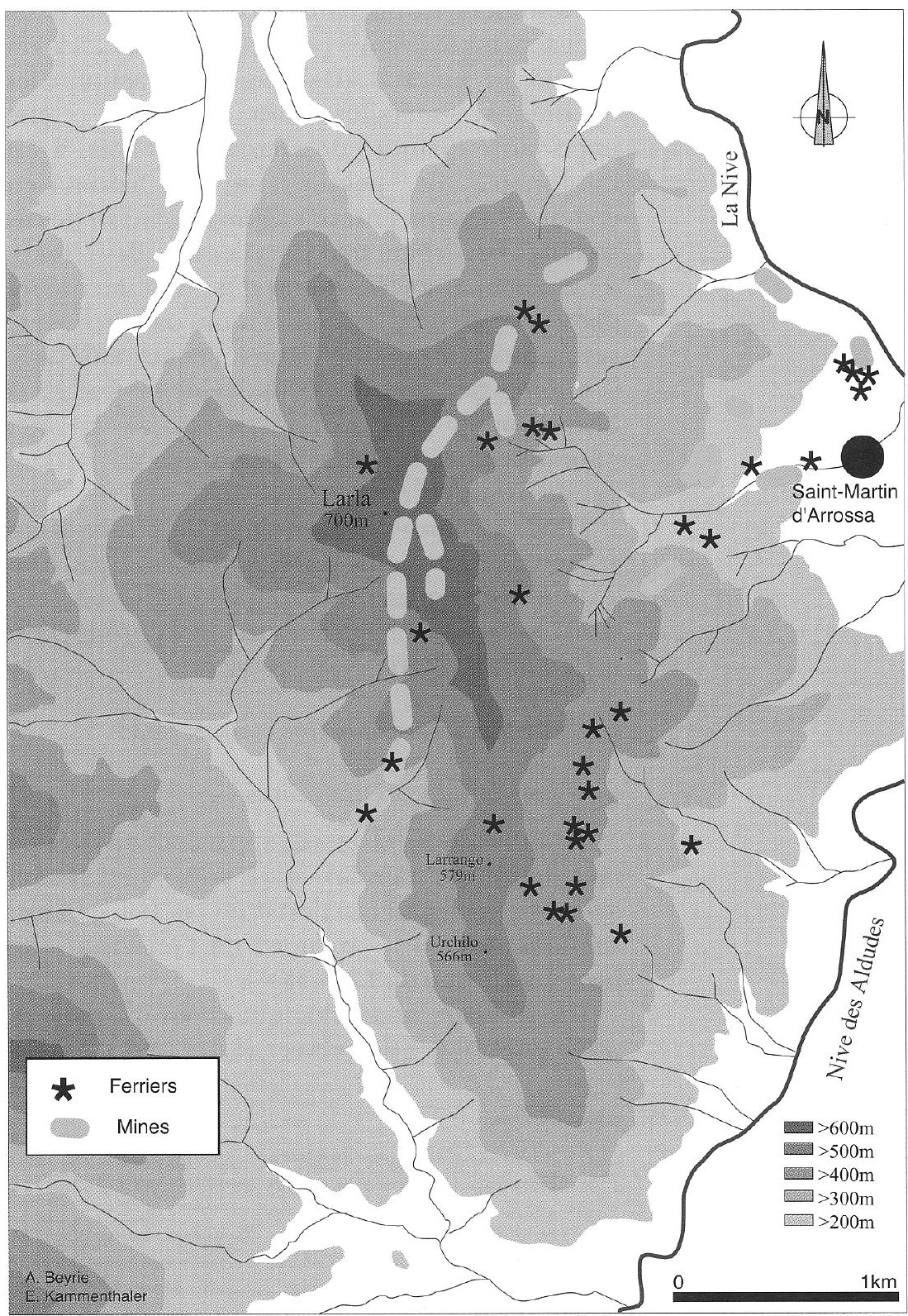

Fig. 6. Vestiges miniers et métallurgiques de la montagne Larla. 
l'activité trahit vraisemblablement plusieurs modes d'exploitation des ressources du massif.

Les sites de l'ensemble nord se trouvent à proximité immédiate du filon dit d'Ustelegi. Ils sont grossièrement alignés dans l'axe de la minéralisation de sidérite, au cœur du district minier majeur. Aucun vestige extractif en revanche n'a été localisé à proximité du groupe métallurgique méridional. Dans ces conditions, soit le minerai extrait dans les parties nord de Larla était acheminé jusqu'à ces ateliers, situés 1 à $2 \mathrm{~km}$ au sud et en aval des sites d'extraction (les opérations métallurgiques exigeant une importante quantité de charbon de bois, il est raisonnable de penser que le besoin en combustible a pu entraîner l'implantation de ces ateliers de réduction à l'écart des zones d'extraction), soit les mines qui ont alimenté les dix-sept ateliers de réduction n'ont pas encore été reconnues. Quant au groupe métallurgique aval, il semble que son développement ait été lié à l'exploitation de filons secondaires, des minéralisations de sidérite jalonnées de vestiges extractifs superficiels ${ }^{23}$.

D'un point de vue chronologique, les sondages archéologiques réalisés sur huit ferriers, quatre du groupe sud et quatre du groupe nord, ont permis d'attester l'antiquité d'une part de la production métallique de Larla ${ }^{24}$.

\section{L'atelier métallurgique Harotzainekoborda}

\section{— Les études préliminaires}

Le site Harotzainekoborda appartient au groupe métallurgique méridional. L'atelier est établi légèrement en aval et à l'est d'un col (alt. 544 m), à moins de $150 \mathrm{~m}$ au nord de l'enceinte dite protohistorique de Larrango ${ }^{25}$.

Un sondage archéologique, conduit dans la partie supérieure du crassier (S.1. : 1,60 x $1 \mathrm{~m}$ ), a

23. Ces filons se distinguent du filon principal dit d'Ustelegi. Il s'agit de minéralisations à quartz et sidérite encaissées dans des terrains paléozoïques.

24. Les datations au radiocarbone effectuées sur les sites de Harotzainekoborda, Pellosastreanekoborda 2, Antxartezaharreborda 1, et Haritzondo, ont révélé une activité comprise entre la deuxième moitié du I ${ }^{\text {er }}$ siècle a.C. et le début du Ive siècle p.C. Les sites Oheta 1 et Larla 1 sont en cours de datation. révélé un amas de déchets métallurgiques peu épais (haut.: 0,5 m), mais probablement largement étalé si l'on en juge par l'épandage de scorie sur les pentes orientales du col. Les enseignements de ce sondage confrontés à la topographie générale du site laissaient pressentir la présence de fourneaux de réduction quelques dizaines de mètres en amont, sur les premières pentes du col.

Le ferrier n'étant pas clairement identifiable dans la configuration topographique environnante, une prospection magnétique a été conduite sur la partie supérieure du col ${ }^{26}$. Elle devait être en mesure de fournir une vision plus claire de l'atelier dans son ensemble, en délimitant précisément les limites du crassier, et surtout en déterminant l'emplacement des bas fourneaux de réduction du centre de production antique. La prospection à vue et l'étude topographique du site ne révélaient en effet aucun indice encourageant des hypothèses archéologiques quant à la localisation possible des fours métallurgiques. L'image géophysique obtenue présentait quatre ensembles caractérisés par des valeurs de susceptibilités magnétiques relativement élevées, anomalies pouvant correspondre à des sources d'origine anthropique liées à l'activité métallurgique. Ces données ont été interprétées en fonction de la topographie du site et des divers indices révélés par la prospection au sol et le sondage d'évaluation archéologique.

\section{— Les opérations archéologiques}

Guidés par la carte géophysique, deux sondages archéologiques ont été entrepris sur le site Harotzainekoborda (S.2. : $4 \mathrm{~m}^{2}$; S.3. : $5,5 \mathrm{~m}^{2}$ ). Ces interventions se sont soldées par la mise au jour d'un crassier et d'une structure de réduction comprenant un bas fourneau (LARLA.F.1) et une fosse de travail. Remarquablement bien conservé,

25. Les sondages archéologiques entrepris à l'intérieur des ouvrages en pierre sèche de Larrango et d'Urchilo ont écarté l'éventualité d'un lien entre l'activité métallurgique antique et les deux sites à enceinte. La datation des fragments de charbon de bois prélevés à Urchilo révèle en outre une activité anthropique datant du XIII ${ }^{e}$ siècle p.C. (LY-10301).

26. La surface couverte par la prospection représente $1750 \mathrm{~m}^{2}$ (Guyot 2001, 13-14). 
Aucun élément de la porte du four n'est conservé entre les piédroits. Elle devait être conçue en argile et certainement détruite, dans sa partie basse au moins, à la fin de chaque opération de réduction pour faciliter le défournement.

Il est difficile d'estimer la hauteur initiale du bas fourneau. Si l'on considère cependant la nature et la quantité des matériaux contenus dans les niveaux d'effondrement du four, il semble que l'élévation composée de dalles de grès et d'argile devait être peu importante.

La morphologie du bas fourneau étant reconnue, il convient d'en restituer le mode opératoire. L'élévation modeste de la structure semi-enterrée devait autoriser un chargement et une alimentation depuis le niveau de sol observé autour du four, sans qu'il ait été besoin d'aménager de plate-forme ou de terrasse surélevée. Le système mis en œuvre pour la ventillation n'est en revanche pas restituable, la structure de réduction conservée ne possédant aucun orifice d'aération. La morphologie du four, avec une cuve largement enterrée, n'exclut pas une ventilation naturelle, et l'implantation de l'atelier sur un col venté devait être favorable à ce procédé. Si l'on envisage, en revanche, l'utilisation de soufflets, l'emplacement des tuyères reste problématique: soit elles étaient aménagées à l'arrière du four, presque au sommet de la cuve, ce qui les place à une grande distance du creuset; soit les tuyères étaient installées à l'avant du four, et les soufflets actionnés depuis la fosse de travail. Cet espace dégagé offrait en outre la possibilité d'accéder aux parties enterrées du four. Il permettait d'effectuer les réparations nécessaires entre deux opérations de réduction (curage de la cuve, reconstruction de la porte et rechapage de l'enduit interne), facilitait l'écoulement de la scorie et l'extraction de la loupe de fer.

\section{CONCLUSION}

Les grandes lignes chronologiques et l'organisation générale des centres de production antiques de Larla et d'Errola sont établies. Si l'étude archéologique des sites miniers fait encore défaut, les méthodes appliquées à l'étude des ateliers métallurgiques ont été validées par la mise au jour de plusieurs vestiges de bas fourneaux, tandis que le mode de fonctionnement des centres de réduction est partiellement reconnu.

L'hypothèse d'une production de fer antique étant attestée en vallée de Baigorri, il reste à évaluer l'importance de cette activité dans le contexte économique local. Le pôle de Larla, qui se distingue par le nombre et la densité de ses vestiges miniers et métallurgiques, peut sans doute être considéré comme un district de production de fer majeur au Pays Basque durant l'Antiquité. Avec les mines de cuivre de Banca, et celles de galène de la forêt d'Haira, il révèle toute l'importance du foyer minier et métallurgique établi en vallée de Baigorri au cours des premiers siècles de notre ère.

La confrontation des données paléométallurgiques et paléoenvironnementales confirme ces considérations. L'approche interdisciplinaire révèle une étroite correspondance entre les évidences archéologiques d'une part, et les résultats des études géochimiques et palynologiques d'autre part. Les vestiges métallurgiques, les signaux de pollution, de même que les indices d'une reprise des déforestations désignent une phase d'activité minière et métallurgique dont le démarrage se situerait à la fin de l'âge du Fer (II siècle a.C.), et le repli au IV siècle p.C. Cette phase s'insère dans un processus d'exploitation de longue durée et ne dépeint qu'une part de l'histoire industrielle de la vallée de Baigorri. Les indices de paléopollution suggèrent en effet l'existence d'une production métallique locale dès l'âge du Bronze moyen, une proto-industrie dont les traces archéologiques font encore défaut. 


\section{BIBLIOGRAPHIE}

Ambert, P. (1997) : "Recherche sur les fours de métallurgistes Chalcolithiques et de l'âge du Bronze d'Europe occidentale (domaines méditerranéen et alpins)", Archéologie en Languedoc, 21, 29-44.

Ancel, B. (2000) : Les mines de cuivre de Banca, SaintÉtienne-de-Bä̈gorry, Pyrénées-Atlantiques, Rapport de fouille, SRA Aquitaine.

Ancel, B., C. Dardignac, G. Parent et A. Beyrie (2001) : "La mine de cuivre des Trois Rois à Banca, vallée de Baigorry (Pyrénées-Atlantiques)", in : Les ressources naturelles des Pyrénées. Leur exploitation durant l'Antiquité, Entretiens d'archéologie et d'histoire $n^{\circ} \%$, Saint-Bertrand-de-Comminges, musée archéologique départemental, 119-139.

Beyrie, A. (2000) : Mines et métallurgies antiques dans les vallées de la Nive et des Aldudes, Rapport de prospection thématique, SRA Aquitaine.

- (2001) : Mines et métallurgies antiques du fer dans les vallées de la Nive et des Aldudes, Rapport de prospection thématique, SRA Aquitaine.

- (2002): "La métallurgie antique du fer en Labourd et en Bassa-Navarre (PyrénéesAtlantiques)", in : Extraction et traitements des minerais, de la protohistoire au XIX $X^{e}$ siècle, Actes du colloque de SaintClément-les-Places (novembre 2001), sous presse.

Bindler ; R., M. J. Brännvall, I. Renberg, O. Emteryd et H. Grip (1999) : "Natural lead concentrations in pristine boreal forest soils and past pollution trends: a reference for critical load models", Environmental Science and Technology, 33, 3362-3367.

Brännvall, M. L., R. Bindler, O. Emteryd, M. Nilsson et I. Renberg (1997): "Stable isotope and concentration records of atmospheric lead pollution in peat and lake sediments in Sweden", Water, Air, and Soil Pollution, 100, 243-252.

Dietrich, (1786) : Description des gites de minerai des forges et des salines des Pyrénées, Paris-Genève.

Dunlap, G. E., E. Steinnes et A. R. Flegal (1999) : "A synthesis of lead isotopes in two millennia of European air", Earth and Planetary Science Letters, 167, 81-88.

Faure, G. (1986) : Principles of isotope geology, 2nd edition.

Galop, D., F. Monna, A. Beyrie, L. Carozza, V. Mougin et G. Parent (sous presse) : "Métallurgie et histoire de l'environnement au cours des cinq derniers millénaires en Pays Basque nord (Vallée de Baigorri, Pyrénées Atlantiques, France): résultats préliminaires d'une approche interdisciplinaire", Archeologia Postmedievale.

Galop, D., M. Tual, F. Monna, J. Dominik, A. Beyrie et F. Marembert (2001): "Cinq millénaires de métallurgie en montagne basque. Les apports d'une démarche intégrée alliant palynologie et géochimie isotopique du plomb”, Sud-Ouest Européen, 11, 3-15.
Gapillou, C. (1995): “Ébauche géologique et métallogénique de la vallée de Baigorri”, in : Machot 1995, 39-57.

Görres, M. et B. Frenzel (1997) : "Ash and metal concentrations in peat bogs as indicators of anthropogenic activity", Water, Air, and Soil Pollution, 100, 355-365.

Goyehenetxe, M. (2001) : Histoire générale du Pays Basque. Evolution économique et sociale du XVtème au XVIII'̀me siècle, Bayonne.

Guilaine, J. (1997) : "Les débuts de la métallurgie du cuivre en Méditerranée occidentale. Questions ouvertes, hypothèses archéologiques", Archéologie en Languedoc, 21, 9-16.

Guyot, I. (2001): Étude Géophysique, Prospection magnétique sur cinq sites des Pyrénées-Atlantiques, Rapport de prospection, SRA Aquitaine.

Hourmat, P. (1995) : "L'exploitation des mines de cuivre de la vallée de Baïgorry au XVIIJ siècle", in : Machot 1995, 59-100.

Kempter, H. et B. Frenzel (1999) : "The local nature of anthropogenic emissions sources on the elemental content of nearby ombrotrophic peat bogs, Vulkaneifel, Germany", The Science of the Total Environment, 241, 117-128.

(2000): "The impact of early mining and smelting on the local tropospheric aerosol detected in ombrotrophic peat bogs in the Harz, Germany", Water, Air and Soil Pollution, 121, 93-108.

Kempter, H., M. Görres et B. Frenzel (1997) : "Ti and $\mathrm{Pb}$ concentrations in rainwater-fed bods in Europe as indicators of past anthropogenic activities", Water, Air, and Soil Pollution, 100, 367-377.

Kramers, J. D. et I. N. Tolstikhin (1997) : "Two terrestrial lead isotope paradoxes, forward transport modelling, core formation and the history of the continental crust", Chemical Geology, 139, 75-110.

Lefèbvre, T. (1933): Les modes de vie dans les Pyrénées atlantiques orientales, Paris.

Machot, P., éd. (1995): Mines et établissements métallurgiques de Banca, Biarritz/Saint-Étienne-deBaigorry.

Machot, P. et G. Parent (2002) : "Mines et métallurgie en vallée de Baïgorry", in : La vallée de Bä̈gorry, SaintÉtienne-de-Baïgorry.

Mackenzie, A. B., E. M. Logan, G. T. Cook et I. D. Pulfrod (1998): "A historical record of atmospheric depositional fluxes of contaminants in west-central Scotland derived from an ombrotrophic peat core", The Science of the Total Environment, 222, 157-166.

Martinez-Cortizas, A., X. Pontevedra-Pombal, J. C. Novoa-Munöz et E. Garcia-Rodeja (1997) : "Four thousand years of atmospheric $\mathrm{Pb}, \mathrm{Cd}$ and $\mathrm{Zn}$ deposition recorded by the ombrotrophic peat bog of Penido Vello (Northwestern Spain)", Water, Air and soil Pollution, 100, 387-403. 
Monna, F., D. Galop, L. Carozza, M. Tual, A. Beyrie, F. Marembert et J. Dominik (à paraître) : "Impact of local early metalworking in the basque country pointed out by geochemical and pollen records in minerotrophic peat bog".

Monna, F., K. Hamer, J. Lévêque et M. Sauer (2000) : " $\mathrm{Pb}$ isotopes as reliable marker of early mining and smelting in the Northern Harz province (Lower Saxony, Germany)", Journal of Geochemical Exploration, 68, 3, 201-210.

Mougin, V. (2001): "Premières données anthracologiques sur la haute vallée des Aldudes", in: Paléoenvironnement et dynamiques de l'anthropisation de la montagne basque, Projet Collectif de Recherche, Rapport intermédiaire, SRA Aquitaine, 59-61.

Palassou (1784) : Essai sur la minéralogie des MontsPyrénées, Paris.

Renberg, I., M. L. Brännvall, R. Bindler et O. Emteryd (2000) : "Atmospheric lead pollution history during four millennia (2000 BC to 2000 AD) in Sweden", Ambio, 29, 150-156.

Rosman, K., W. Chisholm, S. Hong, J.-P. Candelone et C. Boutron (1997) : "Lead from Garthaginian and Roman spanish mines isotopically identified in Greenland ice dated from 600 B.C. to 300 A.D”, Environmental Science and Technology, 31, 3413-3416.
Shotyk, W., P. Blaser, A. Grünig et A. K. Cheburkin (2000): "A new approach for quantifying cumulative, anthropogenic, atmospheric lead deposition using peat cores from bogs: Pbin eight Swiss peat bog profiles", The Science of the Total Environment, 249, 281-295.

Shotyk, W., A. K. Cheburkin, P. G. Appleby, A. Fankhauser et J. D. Kramers (1996): "Two thousand years of atmospheric arsenic, antimony and lead deposition recorded in an ombrotrophic peat bog profile, Jura moutains, Switzerland", Earth and Planetary Science Letters, 145, E1-E7.

Shotyk, W., D. Weiss, P. G. Appleby, A. K. Cheburkin, R. Frei, M. Gloor, S. Reese et W. O. Van der Knaap (1998): "History of atmospheric lead deposition since $12,37014 \mathrm{C}$ yr BP from a peat bog, Jura mountains, Switzerland", Nature, 281, 1635-1640.

Vié, G. (1979): "Les vielles mines basques, les minéralisations de la Nive des Aldudes", Monde et Minéraux, minéralogie et paléontologie, 33, 12-18.

Weiss, D., W. Shotyk, A. K. Cheburkin, M. Gloor et S. Reese (1997): "Atmospheric lead deposition from 12,400 to 2,000 yrs BP in a peat bog profile, Jura mountains, Switzerland", Water, Air, and Soil Pollution, 100, 311-324. 